\title{
Escala de Autopercepção de Harter para Adolescentes: Um Estudo de Validação
}

\author{
Denise Ruschel Bandeira ${ }^{1}$ \\ Universidade Federal do Rio Grande do Sul \\ Adriane Xavier Arteche \\ University of Reading e Universidade Federal do Rio Grande do Sul \\ Caroline Tozzi Reppold \\ Universidade Federal de Ciências da Saúde de Porto Alegre \\ Universidade Federal do Rio Grande do Sul
}

\begin{abstract}
RESUMO - O presente estudo objetivou investigar a validade convergente da escala de Autopercepção de Harter. Foram participantes 291 adolescentes da Região Sul, com idade média de 17 anos, de ambos os sexos e estudantes do Ensino Médio. Os instrumentos utilizados foram a Escala de Autopercepção de Harter para Adolescentes (EAPH-A), a Escala de Auto-Estima de Rosenberg, a Escala Multidimensional de Satisfação de Vida e o Inventário de Depressão Infantil. Os resultados indicam que a EAPH-A apresentou boa qualidade psicométrica. A análise de fidedignidade apontou índices de consistência interna adequados $(\propto=0,60 \mathrm{a} \propto c=0,88)$. A validade de construto foi comprovada por análises de correlação com os demais instrumentos, sendo que todos os índices foram significativos e na direção esperada. A alta correlação entre os instrumentos aponta a necessidade de haver outros estudos que investiguem as dimensões da autopercepção e diferencie esse conceito de outros construtos, como auto-estima, satisfação de vida e autoconceito.
\end{abstract}

Palavras-chave: escala de autopercepção; auto-estima; validação; adolescente.

\section{Harter Self Perception Scale for Adolescents: A validation study}

\begin{abstract}
The present paper's purpose was to investigate the Harter Self-Perception scale thru a convergent validity analysis. The participants were 291 southern adolescents, mean age 17 years, from both sexes and high school students. The instruments were the Harter Self Perception Scale (HSPS-A), the Rosenberg's Self Esteem Scale, the Multidimensional Life Satisfaction Scale and the Children Depression Inventory. The results offered evidence of satisfactory psychometrics characteristics of the HSPS-A. The reliability analysis indicated acceptable coefficients $(\propto c=0.60$ a $\propto=0.88)$. The construct validity was confirmed thru the correlation analysis with the other instruments once all coefficients were significant and in the expected direction. The high correlation among the instruments indicates the need of other research to evaluate the self-perception dimensions and the relations between this and other correlates constructs such as self-esteem, life satisfaction and self-concept.
\end{abstract}

Key words: self-perception scale; self-steem; validation; adolescent.

O interesse pelas autopercepções do self pode ser observado desde a época da filosofia grega (Harter, 1999) e os estudos das atuais conceitualizações acerca da autopercepção podem ser encontrados antes mesmo do século XX, nos trabalhos de James, Adler, Mead e Cooley, por exemplo (Gobbita \& Guzzo, 2002). No entanto, foi durante o último século que esse tema emergiu como um dos construtos centrais da psicologia (Brown \& Marshall, 2001; Greenwald \& cols., 2002; Tafarodi \& Milne, 2002), especialmente por trabalhos acerca da auto-estima.

Ainda hoje, são muitas as divergências acerca das variáveis que se associam à autopercepção do self. Entre as principais controvérsias, destacam-se três. A primeira diz respeito aos termos que designam a autopercepção e suas definições teóricas (auto-estima; auto-representação; auto-conceito; auto-competência...). A segunda, ao modelo explicativo das variáveis: trata-se de um construto multifacetado, composto por diferentes domínios, ou uma medida global? A terceira refere-se à estabilidade da autopercepção, especificamente

1 Endereço: Av. Ramiro Barcelos 2600, sala 120, Santa Cecília, Porto Alegre, RS, Brasil 90035-003. E-mail: drbandei@terra.com.br ao questionamento se esse construto representaria um estado ou um traço de personalidade. Essas diferenças implicam distintas formas de mensuração dessa variável (Nugent, 2004; Robins, Hendin \& Trzesniewski, 2001) e divergências acerca da validade de conteúdo dos instrumentos que se propõem a avaliar o construto autopercepção.

Um dos instrumentos mais utilizados para avaliação da autopercepção do selfé a Escala de Autopercepção de Harter. Harter (1999), em seu livro Definições sobre o Self, indica que a diferença essencial entre os termos que se referem à auto-avaliação do self está na presença ou ausência de julgamento de valor. No entanto, a autora refere que mesmo estudos que focam as autodescrições não devem ser considerados livres de julgamento de valor. Esse ponto de vista é compartilhado por outros autores, que afirmam que embora nomeiem o construto como auto-estima, o descrevem de forma similar ao de autopercepção, dizendo que o mesmo envolve um julgamento de valor afetivo do indivíduo sobre seus predicados pessoais (Bandura, 1997; Bosma, Graafsma, Grotevant \& Levita, 1994).

Greenwald e cols. (2002) definem operacionalmente autoestima como "a associação do conceito sobre o próprio self 
com um atributo de valor sobre o mesmo" (p. 5) e a distinguem de auto-conceito, indicando que este é " a associação do conceito sobre o próprio self com uma ou mais características (sem juízo de valor)" (p. 5). No que diz respeito à auto-estima e autocompetência, Hermann, Leonardelli e Arkin (2002) indicam que esses são construtos distintos. Enquanto a auto-competência refere-se a quanto a pessoa se considera capaz em determinadas habilidades, a auto-estima refere-se ao julgamento global do sujeito sobre si mesmo.

Quanto ao modelo explicativo da autopercepção, Harter (1999) questiona a unidimensionalidade do construto e sua posição é explícita em relação à defesa da multidimensionalidade. A concepção de Harter contrapõe os teóricos que sugerem que a auto-estima é um construto global. Estes indicam que, ao avaliar sua própria auto-estima, a pessoa pondera sentimentos positivos e negativos sobre o self, fazendo uma avaliação geral sobre si (Hermann \& cols., 2002; Robins \& cols., 2001).

Com essas duas formas de compreender a estrutura da auto-estima, foram desenvolvidos diferentes instrumentos para sua avaliação. A Escala de Autopercepção de Harter (1999) é o principal representante da perspectiva multidimensional e a Escala Rosenberg (1979) é o instrumento mais utilizado para avaliação unidimensional, sendo também o mais utilizado em pesquisas acerca da auto-estima (Tafarodi \& Milne, 2002; Whiteside-Mansell \& Corwyn, 2003).

Além da decomposição da auto-estima em diferentes subfatores, alguns autores propõem que essa medida deva ser avaliada em diferentes domínios (social, escolar, familiar, etc...), sugerindo que os sujeitos possam ter percepções positivas de si em algumas áreas e não em outras. Afirmam, ainda, que tais interações são muito complexas para serem avaliadas por uma única medida (Vispoel Boo \& Bleiler, 2001). Entre os expoentes desse modelo, destacam-se Harter (1998) e Coopersmith (1959).

Apesar das diferenças teóricas sobre o caráter global ou muldimensional da auto-estima, percebe-se que tanto a Escala Rosenberg quanto o instrumento de Harter mensuram a auto-estima a partir de indicadores estáveis da personalidade (Block \& Robins, 1993). Em relação à estabilidade da auto-estima, os estudos tendem a relacioná-la com outros aspectos de personalidade, analisando-a, assim, como uma medida estável. Segundo Block e Robins (1993), embora no início da adolescência os indivíduos sejam suscetíveis à maior inconstância dos sentimentos sobre si, a auto-estima tende à estabilidade ao longo do ciclo vital.

Para Harter (1999), é na adolescência que emergem as autodescrições baseadas em abstrações a respeito do self, uma vez que novas habilidades cognitivas são desenvolvidas nessa etapa do desenvolvimento. Além disso, é durante a adolescência que o jovem mostra-se mais preocupado com o que as outras pessoas pensam sobre ele, o que parece levar a uma baixa nos níveis de auto-estima na adolescência inicial e média. Cabe destacar que, durante esse processo, as meninas apresentam mais pontos de conflito do que os meninos, assim como dão mais importância aos relacionamentos interpessoais.

No entanto, a formação dos sentimentos de autovalor é uma função complexa, que envolve aspectos contextuais, de personalidade e emocionais. Como parâmetro de mensuração da auto-estima, pode-se considerar os sentimentos decorrentes de períodos ou situações específicas ou aspectos de personalidade menos transitórios, como autodepreciação e auto-eficácia. Em geral, os estudos que apresentam a auto-estima como um fator estável, analisam determinantes relacionados a variáveis pouco vulneráveis às experiências imediatas. Entre eles, sexo, classe social, habilidades acadêmicas, perdas familiares e eventos de vida.

Watson, Suls e Haig (2002) buscaram investigar se a auto-estima representa um traço de personalidade por meio de um estudo correlacional com os fatores do Modelo dos Cinco Grandes Fatores. Para tanto, conduziram três estudos nos quais aplicaram a Escala Rosenberg de Auto-Estima e o NEOPI-R, um inventário de personalidade para adultos. Os resultados indicaram que a auto-estima parece representar um traço de personalidade, sendo a ponta de um contínuo, que tem no outro extremo, variáveis como a depressão. Contudo, Watson e cols. (2002), com o modelo hierárquico da personalidade, indicam empiricamente que a auto-estima representa um construto de nível mais baixo na estrutura da personalidade e não um grande fator como neuroticismo. Portanto, a associação entre a auto-estima e os grandes fatores de personalidade deve ser discutida de forma cuidadosa, evitando-se análises isoladas de outras dimensões da personalidade.

A partir dessas considerações teóricas a respeito das controvérsias sobre o construto da autopercepção/auto-estima, o presente estudo objetiva investigar a validade convergente da Escala de Autopercepção de Harter por meio de análises de correlação desta com a Escala de Auto-Estima de Rosenberg, com a Escala Multidimensional de Satisfação de Vida e com o Inventário de Depressão Infantil (Hutz, 2000).

\section{Método}

\section{Participantes}

Participaram do presente estudo 291 adolescentes, com idade média de 17 anos $(d p=1,08)$, de ambos os sexos $(48,5 \%$ meninos e 51,5\% meninas), e estudantes do Ensino Médio ( $78 \%$ alunos do $3^{\circ}$ ano). Os participantes eram procedentes da região Sul do país (62,2\% de Santa Catarina e 37,8\% do Rio Grande do Sul).

\section{Instrumentos}

Os instrumentos utilizados foram a Escala de Autopercepção de Harter para Adolescentes, a Escala de Auto-estima de Rosenberg, a Escala Multidimensional de Satisfação de Vida e o Inventário de Depressão Infantil. A seguir serão descritos os instrumentos considerando suas propriedades psicométricas.

\section{Escala de Autopercepção de Harter para Adolescentes (EAPH-A)}

A Escala de Autopercepção de Harter para Adolescentes é uma adaptação da Escala de Autopercepção de Harter para Crianças (Harter, 1985). A versão infantil foi elaborada a fim de avaliar julgamentos referentes a domínios específicos, bem como de obter uma avaliação global da autopercepção. 
Para tanto, foi dividida em cinco percepções: competência escolar, aparência física, aceitação social e comportamento. A versão para adolescentes foi baseada nos mesmos princípios, sendo mantida a avaliação global e acrescentados três novos domínios: trabalho, amizade e namoro. Cada fator é composto por cinco itens e objetivam investigar diferentes aspectos da autopercepção, conforme abaixo descrito:

1. Competência escolar: avalia a percepção do adolescente em relação à sua competência diante das demandas escolares, por exemplo, como é seu desempenho na sala de aula e o quão inteligente ele julga ser.

2. Aceitação social: investiga o grau com que o jovem é aceito por seus pares, o quanto se sente popular, quantidade de amigos e facilidade para se relacionar.

3. Competência atlética: avalia a percepção do jovem acerca de suas habilidades para os esportes, por exemplo, sentimento de quão bom é seu desempenho em esportes e atividades atléticas.

4. Aparência física: verifica o grau de satisfação do adolescente com a sua aparência, se gosta de seu corpo e se o considera bonito.

5. Trabalho: investiga a extensão em que o examinando possui habilidades para o trabalho, se considera que está apto a realizar atividades laborais e sente que possui um bom desempenho em suas tarefas.

6. Namoro: verifica se o adolescente sente-se atraente, consegue envolver-se com quem deseja e sente-se interessante em um encontro.

7. Comportamento: avalia o nível em que o jovem está satisfeito com o seu comportamento, se age de forma correta e evita situações-problema.

8. Amizade: investiga a habilidade para fazer amigos, bem como para compartilhar questões pessoais e segredos.

9. Autopercepção: avalia a extensão em que o adolescente está satisfeito consigo mesmo, com a forma como conduz sua vida e se é, em geral, feliz. Constitui um julgamento global ao invés de uma avaliação por domínios.

Cada item é dividido em duas sentenças (por exemplo: alguns adolescentes vão muito bem na escola, MAS outros adolescentes não vão bem na escola). $\mathrm{O}$ examinando deve escolher primeiro com qual das duas frases mais se identifica e, após, o quanto se identifica (realmente verdadeiro para mim ou parcialmente verdadeiro para mim). A pontuação é feita no formato Likert de quatro pontos, sendo que 1 representa o pior julgamento do self e 4, o melhor. Nos estudos originais de validação (Harter, 1985) as subescalas apresentaram índices de consistência interna variando entre 0,55 e 0,93, havendo uma variação entre amostras diferenciadas (sexo, idade, escolaridade, por exemplo). Estudos preliminares de validação da escala adaptada para o Brasil apresentaram índices semelhantes aos dados americanos para cada subescala. Nessa amostra, os alphas variaram de 0,48 a 0,84 (Bandeira, Reppold, Pflüger \& Facio, 2003)

\section{Escala de Auto-Estima de Rosenberg}

A Escala de Auto-Estima de Rosenberg (Rosenberg, 1979) é um instrumento de auto-relato, composto por 10 itens. A versão adaptada por Hutz (2000) acrescentou um item à escala, que manteve a avaliação como uma medida unidimensional. As respostas ao teste são dadas por um sistema Likert de quatro pontos (1-4), no qual os participantes devem indicar o grau de concordância com a questão descrita. Quanto maior o escore obtido, maior o índice de auto-estima do respondente. Na presente amostra, a escala Rosenberg apresentou $\propto$ de Cronbach $=0,79$.

\section{Escala Multidimensional de Satisfação de Vida Adaptada para Adolescentes (EMSV-A)}

A Escala Multidimensional de Satisfação de Vida utilizada neste estudo é uma adaptação da Escala Multidimensional de Satisfação de Vida Infantil, desenvolvida por Giacomoni (2002), com base na Escala de Satisfação de Vida de Estudantes (Huebner, 1991) e na Escala de Satisfação de Vida Multidimensional de Crianças (Huebner, 1998), que propõe a avaliação da satisfação de vida infantil a partir de cinco domínios: família, amigos, escola, eu-percebido (self) e ambiente. A EMSV-A (Arteche \& Bandeira, 2003) conta com 61 itens, com respostas no sistema Likert de cinco pontos. Os itens são divididos em sete domínios: família, self comparado, amizade, escola, não-violência, self e trabalho, além de uma medida global e de uma medida total, formada pela soma das subescalas. Na presente pesquisa, a EMSV total apresentou índice de consistência interna $\propto=0,92$, os índices das subescalas variaram entre $\propto=0,77$ e $\propto=0,90$.

\section{Inventário de Depressão Infantil (Children's Depression Inventory - CDI)}

O CDI é um instrumento de mensuração das alterações afetivas, elaborado por Kovacs $(1981,1985)$ a partir do Beck Depression Inventory e adaptado por Hutz e Giacomoni (2000). Pode ser utilizado de forma coletiva ou individual na avaliação de crianças e adolescentes dos 7 aos 17 anos, a fim de detectar a presença e severidade de humor deprimido. O CDI é uma medida unifatorial, composta por 27 itens que investigam aspectos da depressão relacionados a questões vegetativas, cognitivas e psicomotoras. Cada item contém três opções de respostas (pontuada como 0,1 ou 2), em que o participante deve assinalar a que melhor descreve seu estado nas últimas duas semanas. Neste estudo, o coeficiente de consistência interna do instrumento foi de 0,82 .

\section{Procedimentos}

Inicialmente, foi realizado contato com escolas estaduais dos dois estados participantes. Após autorização para realização da pesquisa e emissão do consentimento livre e esclarecido, os instrumentos foram administrados em sala de aula, de forma coletiva, por dois assistentes devidamente treinados. Como contrapartida, foram oferecidas palestras sobre desenvolvimento da auto-estima na adolescência para os professores das escolas participantes.

\section{Resultados}

Foram realizadas análises descritivas da EAPH-A, sendo obtidas as médias da escala geral e das subescalas, considerando também sexo e estado. A comparação das médias 
indicou interação significativa entre sexo e estado em duas das subescalas: comportamento e amizade. Tais dados podem ser visualizados na Tabela 1.

Tabela 1. Média e desvio-padrão da escala total e das subescalas, no total, por sexo e por estado.

\begin{tabular}{|c|c|c|c|c|}
\hline & Sexo & Estado & Média & $\begin{array}{l}\text { Desvio- } \\
\text { Padrão }\end{array}$ \\
\hline \multirow{4}{*}{ AE Geral } & \multirow{2}{*}{ Masculino } & RS & 3,01 & 0,05 \\
\hline & & $\mathrm{SC}$ & 2,84 & 0,03 \\
\hline & \multirow{2}{*}{ Feminino } & $\mathrm{RS}$ & 2,85 & 0,04 \\
\hline & & SC & 2,77 & 0,03 \\
\hline \multirow{4}{*}{ AE Comportamento* } & \multirow{2}{*}{ Masculino } & $\mathrm{RS}$ & 3,10 & 0,07 \\
\hline & & $\mathrm{SC}$ & 2,81 & 0,06 \\
\hline & \multirow{2}{*}{ Feminino } & $\mathrm{RS}$ & 2,88 & 0,07 \\
\hline & & SC & 2,87 & 0,05 \\
\hline \multirow{4}{*}{ AE Amizade* } & \multirow{2}{*}{ Masculino } & $\mathrm{RS}$ & 3,11 & 0,09 \\
\hline & & SC & 2,61 & 0,07 \\
\hline & \multirow{2}{*}{ Feminino } & RS & 3,09 & 0,09 \\
\hline & & $\mathrm{SC}$ & 3,00 & 0,07 \\
\hline \multirow{4}{*}{ AE Namoro } & \multirow{2}{*}{ Masculino } & $\mathrm{RS}$ & 2,78 & 0,08 \\
\hline & & $\mathrm{SC}$ & 2,59 & 0,06 \\
\hline & \multirow{2}{*}{ Feminino } & $\mathrm{RS}$ & 2,75 & 0,08 \\
\hline & & $\mathrm{SC}$ & 2,70 & 0,06 \\
\hline \multirow{4}{*}{ AE Aparência } & \multirow{2}{*}{ Masculino } & $\mathrm{RS}$ & 2,76 & 0,10 \\
\hline & & $\mathrm{SC}$ & 2,83 & 0,07 \\
\hline & \multirow{2}{*}{ Feminino } & $\mathrm{RS}$ & 2,56 & 0,09 \\
\hline & & SC & 2,51 & 0,07 \\
\hline \multirow{4}{*}{ AE Atlético } & \multirow{2}{*}{ Masculino } & $\mathrm{RS}$ & 2,79 & 0,09 \\
\hline & & $\mathrm{SC}$ & 2,73 & 0,07 \\
\hline & \multirow{2}{*}{ Feminino } & $\mathrm{RS}$ & 2,34 & 0,09 \\
\hline & & SC & 2,34 & 0,07 \\
\hline \multirow{4}{*}{ AE Trabalho } & \multirow{2}{*}{ Masculino } & $\mathrm{RS}$ & 3,38 & 0,08 \\
\hline & & $\mathrm{SC}$ & 3,21 & 0,06 \\
\hline & \multirow{2}{*}{ Feminino } & $\mathrm{RS}$ & 3,28 & 0,07 \\
\hline & & $\mathrm{SC}$ & 3,15 & 0,06 \\
\hline \multirow{4}{*}{ AE Escola } & \multirow{2}{*}{ Masculino } & $\mathrm{RS}$ & 2,81 & 0,08 \\
\hline & & $\mathrm{SC}$ & 2,72 & 0,06 \\
\hline & \multirow{2}{*}{ Feminino } & RS & 2,69 & 0,07 \\
\hline & & SC & 2,54 & 0,06 \\
\hline \multirow{4}{*}{ AE Percepção } & \multirow{2}{*}{ Masculino } & $\mathrm{RS}$ & 3,20 & 0,08 \\
\hline & & $\mathrm{SC}$ & 3,09 & 0,06 \\
\hline & & RS & 3,00 & 0,08 \\
\hline & & SC & 2,84 & 0,06 \\
\hline & & $\mathrm{RS}$ & 3,15 & 0,08 \\
\hline & Mrascumno & $\mathrm{SC}$ & 2,95 & 0,06 \\
\hline 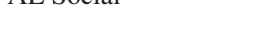 & Faminis & RS & 3,03 & 0,07 \\
\hline & & SC & 2,97 & 0,06 \\
\hline
\end{tabular}

* Interação significativa sexo*estado $\operatorname{com} p<0,05$
Após, foram realizadas análises de consistência interna da escala total e das subescalas. Observou-se que a escala geral apresentou índice de consistência interna muito bom, assim como as subescalas também apresentaram coeficientes aceitáveis, conforme pode ser visualizado na Tabela 2.

Tabela 2. Índices de consistência interna da escala total e das subescalas da Escala de Autopercepção de Harter.

\begin{tabular}{lll}
\hline & Número de itens & $\propto$ de Cronbach \\
\hline Escala Total & 45 & 0,88 \\
AE Aparência & 5 & 0,82 \\
AE Atlético & 5 & 0,80 \\
AE Amizade & 5 & 0,75 \\
AE Percepção & 5 & 0,73 \\
AE Trabalho & 5 & 0,71 \\
AE Escola & 5 & 0,66 \\
AE Comportamento & 5 & 0,64 \\
AE Namoro & 5 & 0,63 \\
AE Social & 5 & 0,60 \\
\hline
\end{tabular}

Por fim, com o objetivo de verificar a validade concorrente da escala, foi realizada a análise de correlação de Spearman com a EMSV-A, com a Escala de Auto-Estima de Rosenberg e com o CDI. Conforme o esperado, as correlações foram positivas e significativas com a EMSV-A e com a Escala de Auto-Estima de Rosenberg. Correlações negativas e significativas foram encontradas com o CDI (ver Tabela 3 ).

Tabela 3. Correlações entre a Escala de Autopercepção de Harter, a Escala Muldimensional de Satisfação de Vida, a Escala de Auto-Estima de Rosenberg e o CDI.

\begin{tabular}{ll}
\hline & Harter \\
\hline EMSV & $0,58^{*}$ \\
Rosenberg & $0,61^{*}$ \\
CDI & $-0,57^{*}$ \\
\hline
\end{tabular}

* correlações significativas com $\mathrm{p}<0,01$

\section{Discussão}

Os resultados indicam que a EAPH-A apresentou boa qualidade psicométrica. A análise de fidedignidade apontou índices de consistência interna adequados, similares aos encontrados na versão original da escala (Harter, 1985). A validade de construto foi comprovada pelas análises de correlação com a Escala de Auto-Estima de Rosenberg, a EMSV-A e o CDI. Todos os índices foram significativos e na direção esperada, evidenciando a qualidade da escala para avaliar autopercepção. A alta correlação entre os instrumentos aponta a necessidade de haver outros estudos que investiguem as dimensões da autopercepção e diferencie esse conceito de outros construtos, tais como auto-estima, satisfação de vida e autoconceito.

A interação de sexo e estado nas subescalas de comportamento e amizade reflete as diferenças biológicas e culturais nos construtos avaliados. De acordo com a literatura sobre o desenvolvimento juvenil, adolescentes do sexo masculino tendem a avaliar de forma mais amena possível 
comportamentos anti-sociais (Patterson, 1998). Por outro lado, meninas valorizam mais que meninos as relações de amizade, resultado já encontrado em estudos com amostras locais (Souza, 2006). O efeito dessa interação mostra que a escala é sensível a esses aspectos demográficos, devendo essas variáveis ser consideradas em futuros estudos de normatização do instrumento.

Ainda que o presente estudo aponte algumas evidências de validade, novos estudos são necessários para que se possa ter maior confiança no uso da escala. São sugeridos estudos com amostras maiores que permitam a realização de análises fatoriais para investigar se as dimensões da autopercepção propostas por Harter (1985) se aplicam à realidade brasileira. Além desses, a realização de pesquisas longitudinais contribuiria para a definição do construto à medida que permitiria a investigação da estabilidade dessa medida e a pertinência dessa como um aspecto da personalidade. Por fim, ressalta-se a necessidade de estudos de validade de critério que apresentem níveis de sensibilidade e especificidade da escala, a fim de que possa ser utilizada clinicamente.

\section{Referências}

Arteche, A. \& Bandeira, D. (2003). Bem-estar subjetivo: Um estudo com adolescentes trabalhadores. Psico-USF, 8(2), 193-202.

Bandeira, D., Reppold, C., Plüger, D. \& Facio, A. (2003). Escala Harter de Auto-Estima: adaptação para o contexto brasileiro. Anais do I Congresso Brasileiro de Avaliação Psicológica e IX Conferência Internacional de Avaliação Psicológica. Campinas: IBAP.

Bandura, A. (1997). Self-efficacy: The exercise of control. New York: W. H. Freeman and Company.

Block, J. \& Robins, R. W. (1993). A longitudinal-study of consistency and change in self-esteem from early adolescence to early adulthood. Child Development, 64, 909-923.

Bosma, H. A., Graafsma, T. L. G., Grotevant, H. D. \& Levita, D. J. (1994). Identity and development: An interdisciplinary approach. California: Sage Publications.

Brown, J. \& Marshall, M. (2001). Self-esteem and emotion: Some thoughts about feelings. Personality and Social Psychology Bulletin, 27(5), 575-584.

Coopersmith, S. (1959). A method for determining types of self-esteem. Journal of Abnormal and Social Psychology, 59, 87-94.

Giacomoni, C. (2002). Bem-estar subjetivo infantil: Conceito de felicidade e construção de instrumentos para avaliação. Tese de Doutorado, Universidade Federal do Rio Grande do Sul, Porto Alegre.

Gobbita, M. \& Guzzo, R. (2002). Estudo inicial do Inventário de Auto-Estima (SEI) Forma A. Psicologia: Reflexão e Crítica, 15(1), 143-150.

Greenwald, A., Rudman, L., Nosek, B., Banaji, M., Farnham, S. \& Mellott, D. (2002). A unified theory of implicit attitudes, stereotypes, self-esteem, and self-concept. Psychological Review, 109(1), 3-25.

Harter, S. (1985). Adolescent Self-Perception Profile. Manuscrito não-publicado.

Harter, S. (1998). The development of self-representation. Em W. Damon (Org. Série) \& N. Eisenberg (Org. Vol.), Handbook of child psychology: Vol. 3. Social, emotional, and personality development. (5 ed., pp. 553-617). New York: John Wiley \& Sons.
Harter, S. (1999). The construction of the self: A developmental perspective. New York: Guilford Press.

Hermann, A., Leonardelli, G. \& Arkin, R. (2002). Self-doubt and Self-esteem: A threat from within. Personality and Social Psychology Bulletin, 28(3), 395-408.

Huebner, E. (1991). Initial development of the student's life satisfaction scale. Correlates of life satisfaction in children. School Psychology International, 12, 229-238.

Huebner, E. (1998). Cross-racial application of a children's multidimensional life satisfaction scale. School Psychology International, 19(2), 179-188.

Hutz, C. S. (2000). Adaptação brasileira da escala de auto-estima de Rosenberg. Manuscrito não-publicado, Curso de PósGraduação em Psicologia do Desenvolvimento, Universidade Federal do Rio Grande do Sul. Porto Alegre, RS.

Hutz, C. S. \& Giacomoni, C. H. (2000). Adaptação brasileira do inventário de depressão infantil (CDI). Manuscrito não-publicado, Curso de Pós-Graduação em Psicologia do Desenvolvimento, Universidade Federal do Rio Grande do Sul. Porto Alegre.

Kovacs, M. (1981). Rating scales to asses depression in school-ages children. Acta Paedopsychiatrica, 46, 305-315.

Kovacs, M. (1985). The Children's Depression Inventory (CDI). Psychopharmacology Bulletin, 21, 995-998.

Nugent, W. (2004). A validity study of two forms os the Self-esteem Rating Scale. Research on Social Work Practice, 14(4), 287-294.

Patterson, G. (1998). Coercion as a basis for early age of onset for arrest. Em J. McCord (Org.), Coercion and punishment in longterm perspectives (pp. 81-105). Cambridge: University Press.

Robins, R., Hendin, H. \& Trzesniewski, K. (2001). Measuring global self-esteem: Construct validation of a single-item measure and the Rosenberg self-esteem scale. Personality and Social Psychology Bulletin, 27(2), 151-161.

Roseenberg, M. (1979). Conceiving the self. New York: Basic Books.

Souza, L. K. (2006). Amizade em adultos: adaptação e validação dos Questionários McGill e um estudo de diferenças de gênero. Tese de Doutorado, Universidade Federal do Rio Grande do Sul. Porto Alegre.

Tafarodi, R. \& Milne, A. (2002). Decomposing global self-esteem. Journal of Personality, 70(4), 443-483.

Vispoel, W., Boo, J. \& Bleiler, T. (2001). Computerized and paper-and-pencil versions of the Rosenberg self-esteem scale: A comparison of psychometric features and respondent preferences. Educational and Psychological Measurement, 61(3), 461-474.

Watson, D., Suls, J. \& Haig, J. (2002). Global self-esteem in relation tu structural models of personality and affectivity, Journal of Personality and Social Psychology, 83(1), 185-197.

Whiteside-Mansell, L. \& Corwyn, R. (2003). Mean and covariance structures analyses: An examination of the Rosenberg selfesteem scale among adolescents and adults. Educational and Psychological Mearument, 63(1), 163-173.

Recebido em 07.08.2006

Primeira decisão editorial em 08.06.2007 Versão final em 08.08.2007 Aceito em 14.01.2008 